\title{
The Kringle-2 domain of tissue plasminogen activator significantly reduces mortality and brain infarction in middle cerebral artery occlusion rats
}

\author{
HAITAO ZHANG ${ }^{1}$, FENG BI $^{2,3}$, CHUNLAN XIAO $^{1}$, JIANXIA LIU ${ }^{3}$, \\ ZHIXIA WANG ${ }^{3}$, JIAN-NING LIU ${ }^{1,2}$ and JING ZHANG ${ }^{1,2}$ \\ ${ }^{1}$ Institute of Molecular Medicine and State Key Laboratory of Pharmaceutical Biotechnology, Nanjing University, \\ Nanjing 210093; ${ }^{2}$ Institute of Molecular and Experimental Therapeutics, East China Normal University, \\ Shanghai 200062; ${ }^{3}$ Landing Bio-pharmaceuticals, Inc., Suzhou 215028, P.R. China
}

Received March 23, 2010; Accepted May 21, 2010

DOI: $10.3892 /$ ijmm_00000456

\begin{abstract}
Tissue plasminogen activator (TPA) showed brainprotective activity within the first 15 min after cerebral ischemia in rats. To understand its molecular mechanism, TPA derivates were intracerebroventricularly administered at 15 min before, and 15, 90,120 min after middle cerebral artery occlusion (MCAO) in rats. The reduction in mortality and cerebral infarction at $24 \mathrm{~h}$ was seen only with TPA administered at 15 min after MCAO. The down-regulation of endogenous TPA by the intracerebroventricular injection of TPA was found to be responsible for the protective effect on the integrity of blood-brain barrier after MCAO, as well as for the reduction in mortality and cerebral infarction. Moreover, for the first time we have found that the Kringle-2 domain is essential for the brain-protective activity of TPA.
\end{abstract}

\section{Introduction}

Currently, tissue plasminogen activator (TPA) is the only thrombolytic agent approved to treat acute ischemic stroke. Because of the considerable risks of cerebral hemorrhage, edema and excitotoxicity (1-3), TPA must be used within $3 \mathrm{~h}$ of the onset of symptoms (4). As a multi-domain serine protease, TPA consists of a finger $(\mathrm{F})$, epidermal growth factor-like (G), two Kringle (K1, K2), and C-terminal serine protease $(\mathrm{P})$ domains. Alteplase is the full-length recombinant human TPA, while reteplase (K2P) is the domain deletion mutant comprising only the Kringle- 2 domain and protease domain of TPA (5).

Correspondence to: Dr Jing Zhang, Institute of Molecular Medicine, Nanjing University, 22 Hankou Road, Nanjing 210093, P.R. China

E-mail: jingzhang2001@gmail.com

Key words: blood-brain barrier, Kringle, middle cerebral artery occlusion, ischemia, stroke, tissue plasminogen activator
After cerebral ischemia, endogenous TPA activates the LRP/NF-кB/MMP-9 pathway (6) and platelet-derived growth factor-CC (PDGF-CC) (7) to increase blood-brain barrier (BBB) permeability, which raises the risk of a haemorrhage in the brain. In pathological conditions, TPA is expressed immediately by neuronal activation (8). Plasmin generated by TPA digests laminin in extracellular matrix to induce neuron death (9). Exogenous TPA injected into the brain increases neuronal damage in TPA knockout mice (10) which are resistant to kainate-induced excitotoxic injury (2). TPA directly cleaves the NR1 subunit of the N-methyl-D-aspartate (NMDA) receptor complex and causes excitotoxicity in neurons (11). TPA also mediates microglial activation in cerebral ischemia via an interaction between its finger domain and annexin II (12).

It is generally accepted that TPA causes brain damage in strokes. However, brain protective effects of TPA have also been reported (13-17). An intracerebroventricular (icv) injection of TPA within 15 min after cerebral ischemia in rats has brainprotective effects independent of its plasminogen activator property (13-15). TPA attenuates zinc-induced cell death through the non-proteolytic function $(15,16)$. The mechanisms of this brain-protective effect are not fully understood.

TPA is also considered as a neuromodulator to regulate the homeostasis of neurovascular units in strokes (18). TPA induces the BBB opening through PDGF-CC $(7,19,20)$, and leads to dose-dependent activity on vascular contractility in cerebral ischemia $(21,22)$.

In the present study, we found that the Kringle-2 domain (residues 176-262) of TPA is the essential structure required for the brain-protective activity of TPA, employing the rat middle cerebral artery occlusion (MCAO) model. The reduction in endogenous TPA by the icv injection of exogenous TPA was found to be responsible for protecting the integrity of $\mathrm{BBB}$ after MCAO, and therefore reduces mortality and cerebral infarction.

\section{Materials and methods}

Materials. Alteplase, reteplase and plasmin were obtained from Genentech (San Francisco, CA) and Sulan Bio-Pharma 
Table I. Primers for semi-quantitative RT-PCR.

\begin{tabular}{|c|c|c|c|c|c|}
\hline Gene & Gene no. & $\begin{array}{l}\text { Primer sequence } \\
\qquad\left(5^{\prime}-3^{\prime}\right)\end{array}$ & Position & $\underset{\left({ }^{\circ} \mathrm{C}\right)}{\mathrm{Tm}}$ & No. \\
\hline $\begin{array}{l}\text { MMP-9 } \\
(1254)\end{array}$ & NM_031055 & $\begin{array}{l}\text { Sense: TTCAAGGACGGTCGGTAT } \\
\text { Antisense: CCTGTGAGTGGGTTGGATT }\end{array}$ & $\begin{array}{l}1609-1621 \\
2844-2862\end{array}$ & 60 & 30 \\
\hline $\begin{array}{l}\text { MMP-9 } \\
(732)\end{array}$ & U36476 & $\begin{array}{l}\text { Sense: CCACTGCACCTCCCATGGCCTATC } \\
\text { Antisense: GCAAAAGAGGAGCCTTAGTT TT }\end{array}$ & $\begin{array}{l}1415-1438 \\
2146-2127\end{array}$ & 58 & 30 \\
\hline NF-кB (p65) & M61909 & $\begin{array}{l}\text { Sense: CGGGGACTATGACTTGAATG } \\
\text { Antisense: ATGGTGCTGAGGGATGTTGA }\end{array}$ & $\begin{array}{c}507-526 \\
1112-1133\end{array}$ & 60 & 30 \\
\hline B-actin & NM_031144 & $\begin{array}{l}\text { Sense: GCCAACCGTGAAAAGATG } \\
\text { Antisense: GCCACCAATCCACACAGA }\end{array}$ & $\begin{array}{c}421-438 \\
1093-1110\end{array}$ & 63 & 25 \\
\hline
\end{tabular}

mRNA of MMP-9 and NF-кB was measured in the ipsilateral ischemic hemisphere using semi-quantitative RT-PCR. B-actin was measured as an internal control.

(Suzhou, China), respectively. Reteplase is a mutant form of TPA which only has Kringle-2 and serine protease domain (P). The isolated Kringle-2 and P of TPA were prepared from reteplase in our laboratory. Plasminogen was purified from rat plasma using lysine-Sepharose 4B (Amersham Pharmacia Biotech, Uppsala, Sweden). Erythrina trypsin inhibitor (ETI)Sepharose was prepared by conjugating ETI to cyanogen bromide activated Sepharose 4B, and was used to purify reteplase.

Preparation of Kringle-2 and $P$ domain of TPA. Kringle-2 and $\mathrm{P}$ were purified as described previously (23), digesting reteplase by plasmin at Arg275-Ile276 (24). Reteplase ( $2 \mathrm{mg} / \mathrm{ml}$ ) was incubated with $2 \mu \mathrm{g} / \mathrm{ml}$ rat plasminogen in the digest buffer $(0.7 \mathrm{~mol} / \mathrm{l} \mathrm{Arg}$ in $\mathrm{PBS}, \mathrm{pH} 8.0)$ at $37^{\circ} \mathrm{C}$ overnight. Reaction mixture was then reduced with dithiothreitol (DTT, $0.5 \mathrm{mmol} / \mathrm{l}$ ) at room temperature for $2 \mathrm{~h}$, and subsequently oxidized with $\mathrm{H}_{2} \mathrm{O}_{2}(0.1 \mathrm{mmol} / \mathrm{l})$ at room temperature for $1 \mathrm{~h}$. After the reaction was terminated by dialysis against the digest buffer, the mixture was applied to an ETI-Sepharose column to isolate the $\mathrm{P}$ domain. The flow-through was dialyzed against $\mathrm{NH}_{4} \mathrm{Ac}(100 \mathrm{mmol} / \mathrm{l}, \mathrm{pH}$ 6.4), and then applied to lysine-Sepharose 4B. The $\mathrm{P}$ and Kringle-2 domain were eluted from the columns and dialyzed against PBS ( $\mathrm{pH} 7.4)$, respectively. Their purities were examined by sodium dodecyl sulfate-polyacrylamide gel electrophoresis (SDS-PAGE). Kringle-2 and $\mathrm{P}$ were filtrated with $0.22-\mu \mathrm{m}$ filter (Millipore, Bedford, MA, USA) before injection.

Experimental model of MCAO in rats. All animal experimental procedures were approved by the Institutional Animal Care and Use Committee of Institute of Molecular Medicine, Nanjing University. As previously described (25), male Sprague-Dawley rats (Qinglongshan, Nanjing, China) weighing 300-320 g were subjected to MCAO for $2 \mathrm{~h}$, and then sacraficed at 3,12 and $24 \mathrm{~h}$ after the initial MCAO. After MCAO (24 h), brains were cut into 2-mm thick coronal sections through matrix (Stoelting, Wood Dale, IL, USA), and stained in isotonic PBS (pH 7.4) containing 2\% triphenyltetrazolium chloride (TTC, Sigma, St. Louis, MO, USA) at $37^{\circ} \mathrm{C}$ for $30 \mathrm{~min}$. Total infarct volumes were calculated with a computer-assisted image analysis and corrected for edema (26).
Effect of TPA derivates on the survival rates and infarction in MCAO rats. After the procedure of MCAO, the rat was mounted on a stereotaxic apparatus (Stoelting, Wood Dale, IL). The test agent $(2.5 \mu 1)$ was icv-injected using a 5- $\mu 1$ Hamilton micro-syringe (Reno, NV) controlled with a Stoelting microsyringe pump (Wood Dale, IL). The micro-syringe was carefully removed $3 \mathrm{~min}$ after injection. At first, four groups of rats were icv injected with 40 pmol of reteplase at $15 \mathrm{~min}$ before and 15, 90, $120 \mathrm{~min}$ after MCAO, respectively. The control group was icv injected with PBS at 120 min after MCAO. Secondarily, at $15 \mathrm{~min}$ after MCAO, five groups of rats were icv-injected with 40 pmol of alteplase, reteplase, Kringle-2, P, plasmin and PBS, respectively. All rats were observed for total $24 \mathrm{~h}$, in which they were ischemic for the first $2 \mathrm{~h}$ and then immediately reperfused for the remaining $22 \mathrm{~h}$. At $24 \mathrm{~h}$, the survival rates were evaluated and the infarct volumes were measured with 2,3,5-triphenyltetrazolium chloride staining (14).

Gene expression of $N F-\kappa B$ and MMP-9 in the brain after $M C A O$. At $24 \mathrm{~h}$ after MCAO, the rat brain was removed. The ischemic hemisphere was then isolated, quickly frozen by liquid nitrogen and stored at $-80^{\circ} \mathrm{C}$. Total RNA of the ischemic hemisphere was prepared with Trizol (Shinegene, Shanghai) and reverse transcribed to cDNA using the Shinegene Firststrand Synthesis Kit (Shinegene, Shanghai). mRNA of MMP-9 and NF- $\mathrm{KB}$ were measured in the ipsilateral ischemic hemisphere using semi-quantitative RT-PCR (6). The NF-кB primer named NF- $\mathrm{KB}$ (p65) and two sets of MMP-9 primers named MMP-9 (1254) and MMP-9 (732) were used in the experiment (Table I). $\beta$-actin was also measured as an internal control. The Bio-Rad Gel Doc 2000 system was applied to image acquisition and analysis.

Zymography assay for endogenous TPA activity in the brain. The endogenous TPA activities in the ipsilateral ischemic hemisphere were measured using casein-zymography as previously reported (27). Briefly, the rat brain was removed and cut into 2-mm thick coronal sections through the matrix. The ipsilateral sides (-2-0 $\mathrm{mm}$ posterior from bregma) were homogenized and centrifuged in a 10-fold volume lysis buffer (50 mmol/1 Tris- $\mathrm{HCl}, 150 \mathrm{mmol} / \mathrm{l} \mathrm{NaCl}, 1 \%$ v/v Triton X-100, 
$\mathbf{A}$

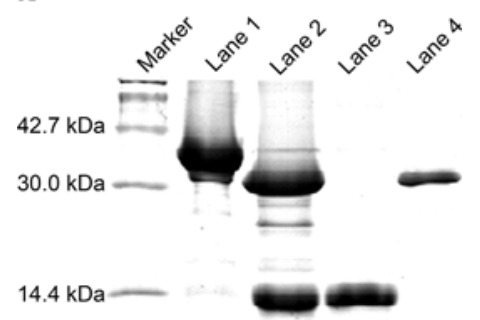

B

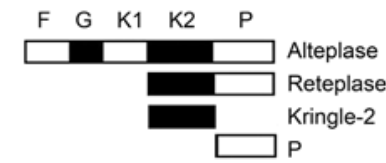

$\mathrm{F}$ : finger domain

G: epidermal growth factor-like domain

K1: Kringle-1 domain

$\mathrm{K} 2$ : Kringle-2 domain

P: protease domain

Figure 1. Purification of Kringle-2 and P domain. Reteplase was digested by plasmin, and then reduced by DTT $(0.5 \mathrm{mmol} / \mathrm{l})$. The product was isolated by ETI- and lysine-Sepharose to obtain P and Kringle-2, respectively. A. Characterization of TPA derivates by SDS-PAGE. Lane 1, reteplase; 2 , reteplase digested by plasmin; 3, Kringle-2 purified by lysine-Sepharose; 4, $\mathrm{P}$ purified by ETI-Sepharose. B. Structure of TPA derivates.

$\mathrm{pH}$ 7.4). The supernatant was obtained to determine the protein concentration and used for SDS-PAGE zymography. Rats were icv-injected with 40 pmol of reteplase at $-15,15$, 90 and 120 min and PBS at 15 min after MCAO, respectively. Rats in every injection group were then sacraficed at 3, 12 and $24 \mathrm{~h}$ following MCAO, respectively. The sham-operation with no MCAO was performed on rats as control.

Assay for BBB permeability after MCAO. The BBB integrity was determined by fluorescence of Evans blue extricated from brain tissue as previously described (28). Evans blue was injected intravenously ( $2 \%$ in saline, $4 \mathrm{ml} / \mathrm{kg}$ body weight) at $5 \mathrm{~h}$ after MCAO. Rats were euthanized at $24 \mathrm{~h}$ after MCAO, and then transcardially perfused with $250 \mathrm{ml}$ heparinized $0.9 \%$ saline, at $110 \mathrm{mmHg}$ pressure. The ischemic hemisections were weighted and homogenized in an 8-fold volume $50 \%$ trichloroacetic acid for $30 \mathrm{sec}$. After centrifugation, the extravasations were diluted with ethanol $(1: 3)$, and the fluorescence was measured by a microplate fluorescence reader (excitation at $620 \mathrm{~nm}$ and emission at $680 \mathrm{~nm}$, Synergy HT). Evans blue at concentration from 50 to $500 \mathrm{ng} / \mathrm{ml}$ was added to the homogenates as standards (29).

Statistical analysis. Survival rates of animals were compared by the $\chi^{2}$ test. Other results were expressed as the means \pm standard error of the mean (SEM). Differences between groups were determined by one-way analysis of variance (ANOVA). The statistical significance was set at $\mathrm{P}<0.01$.

\section{Results}

Isolation of Kringle-2 and P domain of TPA. Reteplase is a bacterially expressed TPA deletion mutant comprising residues 176-527 of native TPA (5). It was used as the initial material to produce the Kringle- 2 and P domain of TPA because of its
Table II. Survival rate $(24 \mathrm{~h})$ of MCAO rats with icv injection of reteplase at different time points (20 rats for each time point).

\begin{tabular}{lccccc}
\hline & PBS & \multicolumn{4}{c}{ Reteplase } \\
\hline $\begin{array}{l}\text { Time point of } \\
\text { icv injection } \\
\text { (min) }\end{array}$ & 120 & -15 & 15 & 90 & 120 \\
$\begin{array}{l}\text { Survival rate } \% \\
(24 \text { h) }\end{array}$ & 70.0 & 70.0 & 90.0 & 65.0 & 60.0 \\
\hline
\end{tabular}

Four groups of rats were icv-injected with 40 picomoles of reteplase at 15 min before and $15,90,120$ min after MCAO, respectively. Control group was icv-injected with PBS at 120 min after MCAO. An increase in thesurvival rate was seen only in the group with an icv injection of reteplase at $15 \mathrm{~min}(\mathrm{P}<0.01, \mathrm{~N}=20)$. There is no SEM presented, since survival rate was analyzed in one research center.

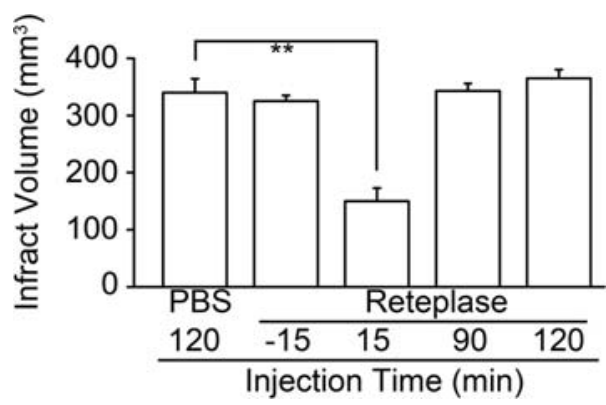

Figure 2. Infarct volume of MCAO rats by icv injection of reteplase. Reteplase was icv-injected at different time points $(-15,15,90$, and $120 \mathrm{~min})$, and PBS was injected at $120 \mathrm{~min}$ after MCAO as control. The lowest infarct volume was found in the $15-\mathrm{min}$ reteplase treated group $(\mathrm{P}<0.01) . \mathrm{n}=20$.

structural simplicity (Fig. 1B). In the reaction system, plasminogen was activated to plasmin by reteplase, and then reteplase was completely cleaved by plasmin into a two-chain form (Fig. 1A, Lane 2), which is composed of Kringle-2 and P domain linked by a disulfide bond. Subsequently the interchain disulfide bond was reduced by $0.5 \mathrm{mmol} / \mathrm{l}$ DTT, leaving the intra-chain disulfide bonds unaffected. Finally, Kringle-2 $(10 \mathrm{kDa})$ and $\mathrm{P}(30 \mathrm{kDa})$ were purified using ETI- and lysineSepharose 4B affinity chromatograph (Fig. 1A, Lanes 3 and 4). The full biological activity of purified Kringle- 2 and $\mathrm{P}$ were recovered, as the denatured protein did not bind to lysine- or ETI- Sepharose 4B (23).

Confirmation of the brain-protective effect of TPA using reteplase in $M C A O$ rats. Rats were divided into four groups with injection of reteplase at different time points. The brainprotective effect of TPA was only found at 15 min after MCAO. As shown in Table II and Fig. 2, the reduction in mortality and cerebral infarct volume at $24 \mathrm{~h}$ were seen only in groups with an icv injection of reteplase at 15 min after MCAO $(\mathrm{P}<0.01)$. This is consistent with previous reports on alteplase (full-length TPA) (13) and its non-protease mutant S478A-TPA $(13,14)$. Interestingly, reteplase administrated at $15 \mathrm{~min}$ before cerebral ischemia had no brain-protective effect. 
Table III. Survival rate ( $24 \mathrm{~h}$ ) of MCAO rats with icv injection of TPA derivates at 15 min after MCAO ( 20 rats per groups).

\begin{tabular}{|c|c|c|c|c|c|}
\hline & \multicolumn{5}{|c|}{ Agent of injection } \\
\hline & PBS & Alteplase & Reteplase & Kringle-2 & $\mathrm{P}$ \\
\hline $\begin{array}{l}\text { Survival rates \% } \\
(24 \mathrm{~h})\end{array}$ & 70.0 & 80.0 & 90.0 & 90.0 & 55.0 \\
\hline
\end{tabular}

At 15 min after MCAO, five groups of rats were icv-injected with 40 pmol of alteplase, reteplase, Kringle-2, P and PBS, respectively. Reteplase and Kringle-2 showed better protective effect than alteplase. $(\mathrm{P}<0.01, \mathrm{n}=20)$. There is no SEM presented, since the survival rate was analyzed in one research center.

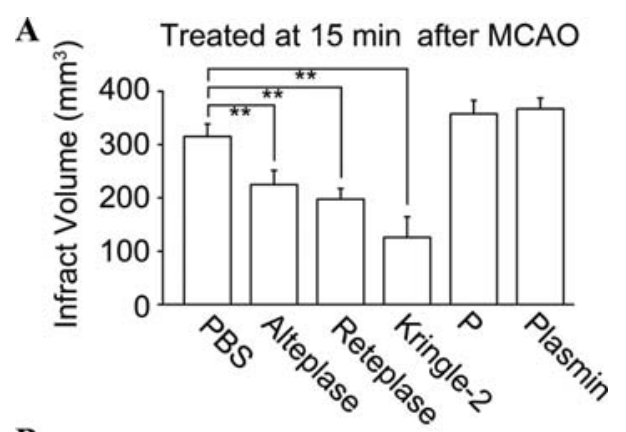

B

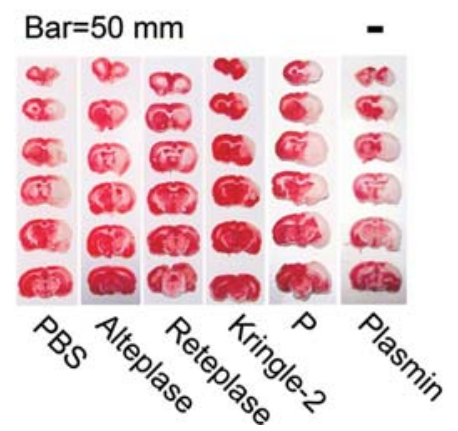

Figure 3. Infarction evaluation of MCAO rats after treatment of TPA derivates. At $15 \mathrm{~min}$ after MCAO, rats were icv-injected with $40 \mathrm{pmol}$ of alteplase, reteplase, Kringle-2, P, plasmin, and PBS, respectively. After $24 \mathrm{~h}$, the brain was stained with 2,3,5-triphenyltetrazolium chloride and the infarction volume was calculated. A. Infarct volumes of PBS, alteplase, reteplase, Kringle-2, P, and plasmin treatment groups at 15 min after MCAO. TPA derivates, including alteplase, reteplase and Kringle-2, significantly reduced infarction volume $(\mathrm{P}<0.01)$. However, $\mathrm{P}$ domain and plasmin had no improvement in infarction. B. Brain sections stained by triphenyltetrazolium chloride. Non-infarction area was stained red, while the infarction area appeared white. $n=20$.

Characterization of Kringle-2 as the structural determinant for the brain-protective effect of TPA in cerebral ischemia. With an icv injection at $15 \mathrm{~min}$ after MCAO, Kringle-2 had the best protective effect in comparison with alteplase and reteplase (Table III, and Fig. 3). In contrast, P and plasmin did not reduce infarction, but caused more death than the PBS controls. This suggests that the protease activity of TPA negatively affects the ischemic brain. The effects of plasminogen Kringle domains were excluded using angiostatin (Kringle 1-4 of plasminogen/plasmin, data not shown). Therefore, the Kringle-2 domain appeared to be the structural determinant of TPA for its protective effect in cerebral ischemia.
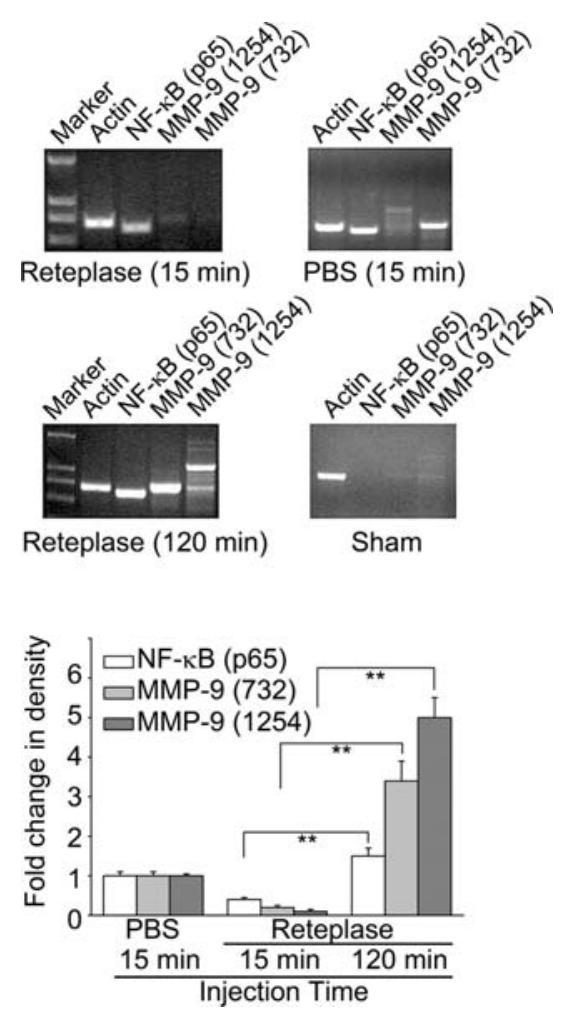

Figure 4. Gene expression of NF-кB and MMP-9 after reteplase treatment. Rats were treated with reteplase at 15 and $120 \mathrm{~min}$, and PBS at $15 \mathrm{~min}$ after MCAO, respectively. The sham-operation was performed as control. The mRNA of MMP-9 and NF-кB were measured in the ipsilateral ischemic hemisphere using semi-quantitative RT-PCR, at $24 \mathrm{~h}$ after MCAO. Three primers named MMP-9 (1254), MMP-9 (732) and NF-кB (p65) were used in the experiment. The cerebral MMP-9 and NF- $\mathrm{B}$ mRNA were significantly down-regulated in rats treated with reteplase at $15 \mathrm{~min}$ than those treated with reteplase at $120 \mathrm{~min}$ and $\mathrm{PBS}$ at $15 \mathrm{~min}(\mathrm{P}<0.01) . \mathrm{n}=10$.

Additionally, reteplase showed a better protective effect than alteplase. This suggests that F, P, or K1 domain in TPA diminishes the brain-protective effect. It was reported previously that the Finger domain of TPA can activate microglial cells and increase brain damage after a stroke (12), and excitotoxic effect of TPA resulted from its proteolytic activity.

Down-regulation of gene expression of $N F-\kappa B$ and $M M P-9$ by reteplase in the brain after $M C A O$. Since NF- $\mathrm{B}$ and MMP-9 expression were induced by TPA via LRP and thereby increased the BBB permeability (6), we investigated their expression by semi-quantitative RT-PCR. The gene expression 


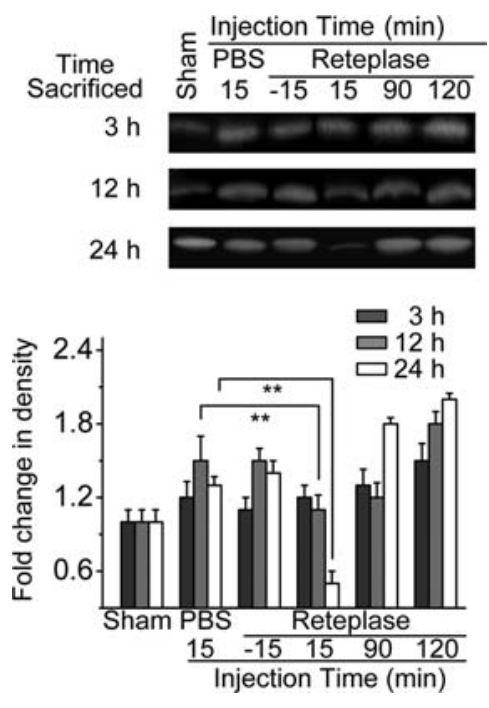

Figure 5. Cerebral endogenous TPA activity after icv injection of reteplase. Rats, which were icv injected with reteplase at $-15,15,90$ and 120 min after MCAO, were sacrificed at 3, 12 and $24 \mathrm{~h}$ after MCAO, respectively. The endogenous TPA activities in the ipsilateral ischemic hemisphere were measured using casein-zymography method and converted into the relative fold changes against the sham group. Reteplase given by icv injection at 15 min significantly down-regulated the cerebral endogenous TPA activity at 12 and $24 \mathrm{~h}$ after MCAO $(\mathrm{P}<0.01) . \mathrm{n}=6$.

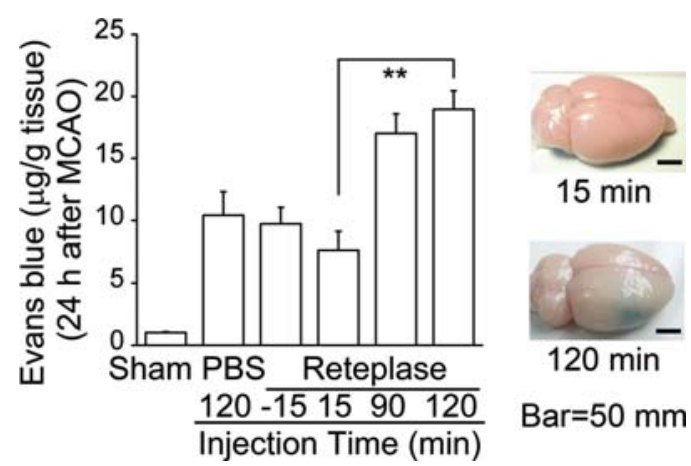

Figure 6. Reduction in BBB permeability after the reteplase treatment. At $24 \mathrm{~h}$ after $\mathrm{MCAO}$, the $\mathrm{BBB}$ integrity was determined by Evans blue staining. Consistent to the changes in endogenous TPA, the BBB permeability of the rats treated at $15 \mathrm{~min}$ was significantly lower than that treated at $120 \mathrm{~min}$ $(\mathrm{P}<0.01)$. $\mathrm{N}=20$.

was expressed as a fold change against the level of the PBS group. The cerebral MMP-9 and NF-kB (p65) mRNA were significantly down-regulated in rats treated with reteplase at 15 min than those treated at $120 \mathrm{~min}$ and the PBS controls $(\mathrm{P}<0.01)$ (Fig. 4).

Down-regulation of cerebral endogenous TPA activity by icv injection of reteplase. Using SDS-PAGE casein-zymography, reteplase $(40 \mathrm{kDa})$ and endogenous TPA $(72 \mathrm{kDa})$ were distinguished based on their molecular weights. Thereby, the cerebral endogenous TPA activity was measured after treatment of reteplase at different time points and expressed as a relative ratio to the sham group. Reteplase icv-injected at 15 min significantly reduced the cerebral endogenous TPA activity at 12 and $24 \mathrm{~h}$ after $\mathrm{MCAO}(\mathrm{P}<0.01)$, while no significant changes were found in the other treatment groups (Fig. 5).

Reduction in BBB permeability by icv injection of reteplase. Two groups of rats were icv injected with reteplase at 15 and 120 min after MCAO, respectively. BBB permeability at $24 \mathrm{~h}$ after MCAO was compared. Rats treated at $15 \mathrm{~min}$ had obviously lower BBB permeability than those treated at 120 min $(\mathrm{P}<0.01)$ (Fig. 6). These data are consistent with the findings of the cerebral endogenous TPA activity shown above.

\section{Discussion}

Consistent with previous finding using the full-length TPA (alteplase or S478A-TPA) (14), we found that the protective effect only occurred at 15 min after MCAO with three TPA derivates, including alteplase, reteplase and Kringle-2. To the best of our knowledge, we have identified for the first time that the Kringle-2 domain is the essential structure required for the brain-protective activity of TPA. Interestingly, the protease domain of TPA actually has an adverse effect and causes brain damage. A non-proteolytic TPA mutant (S478A) was previously found also to reduce infarction in ischemic stroke (14).

Many efforts have been made previously to investigate the mechanisms of the brain-protective effect in the early time of cerebral ischemia. These findings include the non-proteolytic effect of TPA in zinc neurotoxicity (15) and modulation of vascular contractility in cerebral ischemia $(21,22)$. Endogenous TPA was repeatedly reported to be elevated after ischemic stroke (30). We found that icv-injected reteplase at $15 \mathrm{~min}$ after MCAO significantly down-regulated the endogenous TPA activity in brain measured at $24 \mathrm{~h}$ and thereby reduced BBB permeability. It was reported previously that PDGF-CC activated by endogenous TPA increased BBB permeability and brain hemorrhage (7), and therefore icv-injection of reteplase at $15 \mathrm{~min}$ after $\mathrm{MCAO}$ may indirectly inhibit PDGF-CC activation. It is unknown whether the reduction in endogenous TPA by exogenous reteplase is due to the increase in clearance or decrease in expression or secretion of endogenous TPA, and why the effect is only observed within 15 min after MCAO. However, the phenomenon is well correlated to the clinical experience that TPA must be used as early as possible ( $\leq 3 \mathrm{~h})$ to treat ischemic stroke. It seems that exogenous TPA in brain triggers certain mechanisms to reduce the level of endogenous TPA for at least $24 \mathrm{~h}$ via its non-proteolytic Kringle-2 domain at the early time of ischemic stroke. This unknown mechanism seems to only work for a short period of time immediately after stroke, which makes late icv-injection of exogenous TPA useless. TPA is then cleared rapidly in the brain, evidenced by the finding that the injection of reteplase at $15 \mathrm{~min}$ prior to MCAO did not affect any experimental observations.

The cerebral MMP-9 and NF-кB (p65) mRNA were found to be significantly down-regulated in rats treated with reteplase at $15 \mathrm{~min}$ than those treated at $120 \mathrm{~min}$ and controls (Fig. 4). This confirms the finding that reduction in endogenous TPA by exogenous reteplase given at $15 \mathrm{~min}$ after MCAO. TPA can attenuate oxidative stress in cerebral ischemia (15), 
and oxidative stress related NF-кB expression would be accordingly decreased. It was also reported previously that TPA induced the expression of NF-кB and MMP-9 via LRP and thereby increases BBB permeability (6). Therefore, less activation of the TPA/NF-кB/MMP-9 pathway also accounted for the brain-protective effect of TPA by icv-injection at 15 min after cerebral ischemia.

The brain-protective activity of TPA was affected by the administration route. The brain-protective effect of TPA was only found in icv-injection rather than intravenous injection (data not shown). This was consistent with a previous study (14), perhaps because the receptor of TPA/Kringle-2 was located in parenchyma. Unlike plasmin and angiostatin, a number of lysine-binding Kringles, did not have the same brain-protective effect as TPA. This indicates that the effect of Kringle- 2 of TPA was unaffected by plasminogen or plasmin.

In conclusion, we have confirmed that TPA was brainprotective within the first $15 \mathrm{~min}$ after cerebral ischemia in rats. For the first time, the Kringle-2 domain is identified to be essentially required for the brain-protective activity of TPA. The reduction in endogenous TPA by icv injection of TPA is responsible for the protective effect on the integrity of $\mathrm{BBB}$ after MCAO. This finding would be helpful to understand the mechanism of the brain-protective effect of TPA and to develop a new agent to treat ischemic stroke.

\section{Acknowledgements}

The work was supported in part by The Ministry of Science and Technology of China (2009ZX09102-228) and a grant from Landing Bio-pharmaceuticals (Suzhou, China).

\section{References}

1. Lyden PD, Zivin JA, Clark WA, et al: Tissue plasminogen activator-mediated thrombolysis of cerebral emboli and its effect on hemorrhagic infarction in rabbits. Neurology 39: 703-708, 1989.

2. Tsirka SE, Gualandris A, Amaral DG and Strickland S: Excitotoxin-induced neuronal degeneration and seizure are mediated by tissue plasminogen activator. Nature 377: 340-344, 1995.

3. Delzoppo GJ, Zeumer H and Harker LA: Thrombolytic therapy in stroke - possibilities and hazards. Stroke 17: 595-607, 1986.

4. Marler JR, Brott T, Broderick J, et al: Tissue-plasminogen activator for acute ischemic stroke. N Engl J Med 333: 1581-1587, 1995.

5. Hu CK, Kohnert U, Wilhelm O, Fischer S and Llinas M: Tissue-type plasminogen activator domain-deletion mutant BM 06.022: Modular stability, inhibitor binding, and activation cleavage. Biochemistry 33: 11760-11766, 1994.

6. Wang X, Lee SR, Arai K, et al: Lipoprotein receptor-mediated induction of matrix metalloproteinase by tissue plasminogen activator. Nat Med 9: 1313-1317, 2003.

7. $\mathrm{Su}$ EJ, Fredriksson L, Geyer M, et al: Activation of PDGF-CC by tissue plasminogen activator impairs blood-brain barrier integrity during ischemic stroke. Nat Med 14: 731-737, 2008.

8. Qian Z, Gilbert ME, Colicos MA, Kandel ER and Kuhl D: Tissue-plasminogen activator is induced as an immediate-early gene during seizure, kindling and long-term potentiation. Nature 361: 453-457, 1993.

9. Indyk JA, Chen ZL, Tsirka SE and Strickland S: Laminin chain expression suggests that laminin-10 is a major isoform in the mouse hippocampus and is degraded by the tissue plasminogen activator/plasmin protease cascade during excitotoxic injury. Neuroscience 116: 359-371, 2003.
10. Wang YF, Tsirka SE, Strickland S, Stieg PE, Soriano SG and Lipton SA: Tissue plasminogen activator (TPA) increases neuronal damage after focal cerebral ischemia in wild-type and TPA-deficient mice. Nat Med 4: 228-231, 1998.

11. Nicole O, Docagne $\mathrm{F}$, Ali C, et al: The proteolytic activity of tissue-plasminogen activator enhances NMDA receptormediated signaling. Nat Med 7: 59-64, 2001.

12. Siao CJ and Tsirka SE: Tissue plasminogen activator mediates microglial activation via its finger domain through annexin II. J Neurosci 22: 3352-3358, 2002.

13. Kilic E, Hermann DM and Hossmann KA: Recombinant tissue plasminogen activator reduces infarct size after reversible thread occlusion of middle cerebral artery in mice. Neuroreport 10 : 107-111, 1999.

14. Yi JS, Kim YH and Koh JY: Infarct reduction in rats following intraventricular administration of either tissue plasminogen activator (TPA) or its non-protease mutant S478A-TPA. Exp Neurol 189: 354-360, 2004.

15. Kim YH, Park JH, Hong SH and Koh JY: Nonproteolytic neuroprotection by human recombinant tissue plasminogen activator. Science 284: 647-650, 1999.

16. Siddiq MM and Tsirka SE: Modulation of zinc toxicity by tissue plasminogen activator. Mol Cell Neurosci 25: 162-171, 2004.

17. Lee HY, Hwang IY, Im H, Koh JY and Kim YH: Nonproteolytic neurotrophic effects of tissue plasminogen activator on cultured mouse cerebrocortical neurons. J Neurochem 101: 1236-1247, 2007.

18. Benchenane K, Lopez-Atalaya JP, Fernandez-Monreal M, Touzani $\mathrm{O}$ and Vivien D: Equivocal roles of tissue-type plasminogen activator in stroke-induced injury. Trends Neurosci 27: 155-160, 2004.

19. Yepes M, Sandkvist M, Moore EG, Bugge TH, Strickland DK and Lawrence DA: Tissue-type plasminogen activator induces opening of the blood-brain barrier via the LDL receptor-related protein. J Clin Invest 112: 1533-1540, 2003.

20. Fredriksson L, Li H, Fieber C, Li X and Eriksson U: Tissue plasminogen activator is a potent activator of PDGF-CC. EMBO J 23: 3793-3802, 2004.

21. Nassar T, Akkawi S, Shina A, et al: In vitro and in vivo effects of TPA and PAI-1 on blood vessel tone. Blood 103: 897-902, 2004.

22. Armstead WM, Cines DB and Higazi AAR: Plasminogen activators contribute to impairment of hypercapnic and hypotensive cerebrovasodilation after cerebral hypoxia/ischemia in the newborn pig. Stroke 36: 2265-2269, 2005.

23. Carroll VA, Leonid LN, Roy B and Adrian LH: Antiangiogenic activity of a domain deletion mutant of tissue plasminogen activator containing kringle 2. Arterioscler Thromb Vasc Biol 25: 736-741, 2005.

24. Lijnen HR, Nelles L, Van Hoef B, De Cock F and Collen D: Biochemical and functional characterization of human tissuetype plasminogen activator variants obtained by deletion and/or duplication of structural/functional domains. J Biol Chem 265: 5677-5683, 1990.

25. Longa EZ, Weinstein PR, Carlson S and Cummins R: Reversible middle cerebral artery occlusion without craniectomy in rats. Stroke 20: 84-91, 1989.

26. Bederson JB, Pitts LH, Germano SM, Nishimura MC, Davis RL and Bartkowski HM: Evaluation of 2,3,5-triphenyltetrazolium chloride as a stain for detection and quantification of experimental cerebral infarction in rats. Stroke 17: 1304-1308, 1986.

27. Yepes M, Sandkvist M, Wong MKK, et al: Neuroserpin reduces cerebral infarct volume and protects neurons from ischemiainduced apoptosis. Blood 96: 569-576, 2000.

28. Belayev L, Busto R, Zhao W and Ginsberg MD: Quantitative evaluation of blood-brain barrier permeability following middle cerebral artery occlusion in rats. Brain Res 739: 88-96, 1996.

29. Aoki T, Sumii T, Mori T, Wang X and Lo EH: Blood-brain barrier disruption and matrix metalloproteinase- 9 expression during reperfusion injury: Mechanical versus embolic focal ischemia in spontaneously hypertensive rats. Stroke 33: 2711-2717, 2002.

30. Ahn MY, Zhang ZG, Tsang W and Chopp M: Endogenous plasminogen activator expression after embolic focal cerebral ischemia in mice. Brain Res 837: 169-176, 1999. 